\title{
Role of interleukin 6 as a predictive factor for a severe course of Covid-19: retrospective data analysis of patients from a long-term care facility during Covid-19 outbreak
}

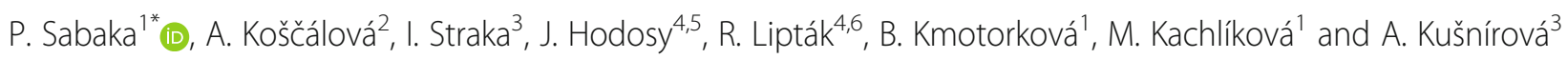

\begin{abstract}
Background: Covid-19 is a disease with high morbidity and mortality among elderly residents of long-term care facilities (LTCF). During an outbreak of SARS-CoV-2 infection in the LTCF an effective screening tool is essential to identify the patients at risk for severe disease. We explored the role of interleukin 6 (IL-6) as a predictor for severe disease during the outbreak of Covid-19 in one LTCF in Slovakia.

Methods: We conducted a retrospective data analysis of cases of COVID-19, diagnosed during the outbreak in one LTCF in Slovakia between April 11, 2020, and May 5, 2020. Within $24 \mathrm{~h}$ after the diagnosis of Covid-19, clinical and laboratory screening was performed in the LTCF to identify patients in need of hospitalization. Patients with oxygen saturation below $90 \%$ were immediately referred to the hospital. Patients staying in the LFTC were monitored daily and those that developed hypoxemia were transferred to the hospital. We analyzed the association between the IL6 at the initial assessment and development of hypoxemia during follow up and determined the cut-off of the IL- 6 able to predict the development of hypoxemia requiring oxygen therapy.
\end{abstract}

Results: Fifty-three patients (11 men, 42 women) with diagnosed Covid-19 were included in the analysis. 19 (53\%) patients developed hypoxemia during the disease. Patients with hypoxemia had significantly higher concentrations of IL-6, C-reactive protein, procalcitonin, fibrinogen, total bilirubin, aspartate aminotransferase and alanine aminotransferase at initial screening. ROC analyses identified IL-6 as the most robust predictor of hypoxemia. The concentration of $\mathrm{IL}-6>24 \mathrm{pg} / \mathrm{mL}$ predicted the development of hypoxemia with the sensitivity of $100 \%$ and specificity of $88.9 \%$. The positive and negative predictive values were 76.9 , and $100 \%$ respectively.

(Continued on next page)

\footnotetext{
* Correspondence: petersabaka@gmail.com

'Department of Infectology and Geographical Medicine, Faculty of Medicine, Comenius University in Bratislava, Bratislava, Slovakia

Full list of author information is available at the end of the article
}

(C) The Author(s). 2021 Open Access This article is licensed under a Creative Commons Attribution 4.0 International License, which permits use, sharing, adaptation, distribution and reproduction in any medium or format, as long as you give appropriate credit to the original author(s) and the source, provide a link to the Creative Commons licence, and indicate if changes were made. The images or other third party material in this article are included in the article's Creative Commons licence, unless indicated otherwise in a credit line to the material. If material is not included in the article's Creative Commons licence and your intended use is not permitted by statutory regulation or exceeds the permitted use, you will need to obtain permission directly from the copyright holder. To view a copy of this licence, visit http://creativecommons.org/licenses/by/4.0/ The Creative Commons Public Domain Dedication waiver (http://creativecommons.org/publicdomain/zero/1.0/) applies to the data made available in this article, unless otherwise stated in a credit line to the data. 
(Continued from previous page)

Conclusions: The concentration of IL-6 $>24 \mathrm{pg} / \mathrm{mL}$ at initial assessment predicted the development of hypoxemia requiring hospitalization with excellent sensitivity and good specificity. IL-6 appears as a potential predictor for the development of the severe Covid-19 and might serve for early identification of patients in need of hospitalization. Further studies are needed to evaluate the robustness of the use of IL-6 as an effective screening tool for the severe course of Covid-19.

Keywords: Covid-19, Long-term care facility, Interleukin 6, Hypoxemia

\section{Background}

Coronavirus disease 2019 (Covid-19) is a disease caused by the zoonotic novel Coronavirus called SARS-CoV-2 [1]. Covid-19 quickly spread across the globe from a place of its origin in Hubei China and pandemic was declared by WHO on March 11, 2020 [2]. Most patients with Covid-19 experience mild self-limiting disease. However, up to $20 \%$ of known cases of Covid-19 are complicated by severe pneumonia which might result in acute respiratory distress syndrome (ARDS) which causes acute hypoxemic respiratory failure [1, 3]. The overall infection fatality ratio is estimated to be below $1 \%$ [4]. It should be noted that fatal outcome may occur at any age including children and young adults [5]. Also, physiologic condition, pregnancy is associated with higher risk of severe disease [6]. However, the risk of death in Covid-19 is increasing with age and a presence of comorbidities, especially cardiovascular diseases, diabetes mellitus and obesity [3]. According to data from the early China epidemic, the case-fatality ratio in the patients over 80 is up to $15 \%$ [7]. Elderly and highly comorbid residents of long-term care facilities (LTCF) are at high risk of Covid-19 associated morbidity and mortality [8-10]. In one well-documented outbreak in LTCF in Washington, USA, more than half of the infected residents required hospitalization and more than one third died [10]. On the background of the ongoing pandemic, the spreading of Covid-19 in LTCF might significantly burden the local health care system and markedly contributes to mortality. Timely and effective intervention is essential to reduce morbidity and mortality during the Covid-19 outbreak in the LTCF. Such intervention consists of quick identification of cases, immediate introduction of infection control measures, initial triage, and daily monitoring of patients. An effective screening tool is essential to identify the patients at risk for severe illness and death [11]. Such patients need close monitoring and early transfer to the hospital. Various biomarkers, especially inflammatory markers like C-reactive protein (CRP), ferritin, fibrinogen, D-dimer and Interleukin 6 (IL-6) are associated with Covid-19 progression [12, 13]. According to known evidence, IL- 6 is superior to CRP and other markers of inflammation in predicting respiratory failure in Covid-19 [14, 15]. IL-6 appears to be the most important driver of immune dysregulation and ARDS in Covid-19 [16-19]. The role of systematic measurement of IL-6, CRP, and other markers of inflammation at the initial assessment during Covid-19 outbreak in LTCF and its ability to predict the severe course of disease is yet to be determined.

\section{Methods}

We conducted a retrospective data analysis of laboratory-confirmed cases of COVID-19, diagnosed during the outbreak in one LTCF in Slovakia between April 11, 2020, and May 5, 2020. A confirmed case of COVID-19 was defined as a positive result on real-time reverse-transcriptase-polymerase-chain-reaction assay of nasopharyngeal swab specimens for SARS-CoV-2. In our analysis, we aimed to identify laboratory markers predicting the severe course of the disease at the initial assessment.

\section{Intervention}

Within 24h after the diagnosis of COVID-19, clinical and laboratory screening was performed in the LTCF by trained clinicians to identify patients in need of hospitalization. The clinical initial assessment consisted of measurement of vital signs (blood pressure, heart rate respiratory rate) and measurement of oxygen saturation by pulse oximetry. Additionally, the venous blood was drawn to measure concentrations of serum glucose, creatinine, urea, sodium, potassium, bilirubin, alanine aminotransferase (ALT), aspartate aminotransferase (AST), CRP, D-dimer, IL-6 and complete blood count. The blood samples were immediately transported to laboratory. The results were obtained and evaluated from 4 to $6 \mathrm{~h}$ after last blood drawn. After an initial assessment, daily monitoring consisting of measurement of vital signs and measurement of oxygen saturation by pulse oximetry was performed by the same clinician. The development of hypoxemia with oxygen saturation below 90\% was considered as the criteria for severe disease with the need for hospitalization. Patients with oxygen saturation below $90 \%$ at the initial screening or during the daily monitoring were immediately referred to the hospital. 


\section{Biochemical analysis}

Concentrations of serum glucose, creatinine, urea, sodium, potassium, bilirubin, ALT, AST, were measured using spectrophotometry (Cobas Integra 400, Roche Diagnostics, Rotkreuz, Switzerland). CRP and D-dimer were measured using immunoturbidimetry (Cobas Integra 400, Roche Diagnostics, Rotkreuz, Switzerland). Serum IL-6 concentrations were measured using an immunoassay (Elecsys, Roche Diagnostics, Rotkreuz, Switzerland).

\section{Statistical analysis}

Quantitative variables are expressed as medians and 25 th and 75 th percentiles. Data in our cohort according to the Kolmogorov-Smirnov test were non-parametric. Medians of quantitative variables between groups were compared using the Mann-Whitney nonparametric test. Effect sizes were assessed using Cohen's d. Receiver operating characteristic curve (ROC) analysis was used to compare markers of hypoxemia. Only the biomarkers with different medians between groups were evaluated in ROC analysis. Optimal cut off values were also determined using ROC analysis. Sensitivity was calculated as the number of true positive divided by true positive + false negative. Specificity was calculated as the number of true negative divided by true negative + false positive. Association of baseline serum concentration of IL-6 $>24$ $\mathrm{pg} / \mathrm{mL}$ with the probability of development of hypoxemia requiring oxygen therapy (HRO) was assessed using multivariate logistic regression. For statistical analysis, SPSS version 26 (International Business Machines Corporation, Armonk, NY, USA) was used.

\section{Ethics}

This study was carried out in concordance with The Code of Ethics of the World Medical Association (Declaration of Helsinki) for experiments involving humans and was approved by the local Ethical Committee of University Hospital Bratislava. The written informed consent was obtained from each participant before enrolment. The investigators preserved the full anonymity of all participants.

\section{Results}

During the intervention in the LTCF, 78 subjects were screened for COVID-19 and 59 patients (47 women, 12 men) had positive pharyngeal swabs for SARS-CoV-2 RNA by RT-PCR and were diagnosed with Covid-19. Fifty-three patients $(90 \%)$ had blood sampling during the initial screening phase. In 6 patients, blood sampling was not performed because of technical difficulties. These patients were excluded from the study. In 53 patients (11 men, 42 women) in which blood sampling was performed, the clinical variables and the results of biochemical and blood count analysis were available for evaluation and were included in the study. Seven patients ( 1 man, 6 women) were diagnosed with severe disease in initial triage and were immediately transferred to a hospital within the first 2 days of intervention. Remaining 45 patients (10 men, 36 women) remained under observation in LTCF and were screened for severe disease and triaged on a daily basis by the intervention team. Overall, 32 patients (6 men, 26 women) were admitted. Among these patients, 19 ( 2 men, 17 women) had hypoxemia required oxygen therapy, 16 patients (2 men, 14 women) were admitted to ICU, and 13 patients ( 2 men, 12 women) died. In 11 patients (1 man, 10 women) initially suffering from hypoxemia requiring oxygen therapy, the disease progressed, and they required intubation and mechanical ventilation. All these patients died because of severe ARDS. One male, initially suffering from hypoxemia requiring oxygen therapy died because of severe gastrointestinal bleeding from gastric adenocarcinoma. One female, initially suffering from hypoxemia requiring oxygen therapy suddenly died after short period of clinical improvement. An autopsy revealed pulmonary embolism. In hospitalized patients, various severe complications were observed. Six patients developed deep vein thrombosis, 6 developed congestive heart failure, 3 patients developed new atrial fibrillation and 5 developed acute renal failure. Baseline characteristics of patients are provided in Table 1. Major clinical findings of all enrolled patients are provided in supplementary table. The baseline serum concentrations of IL6, CRP, procalcitonin, urea, creatinine, fibrinogen, AST, ALT, fasting glucose, total bilirubin and neutrophil count were significantly higher, and lymphocyte and eosinophil count was significantly lower in patients who developed hypoxemia. All patients included in our study seroconverted and developed IgG antibodies within 1 month of diagnosis. The median of baseline serum concentrations of D-dimer and ferritin and a median total count of leukocytes were not significantly different among groups of patients with and without hypoxemia requiring oxygen therapy. Patients who developed hypoxemia requiring oxygen therapy were also significantly more comorbid according to the higher median Charlson Comorbidity Index (Table 1$)$. In the ROC analysis, IL-6 was identified as more robust marker of hypoxemia development than CRP, procalcitonin, CRP, fibrinogen, ALT, AST and total bilirubin and lymphocyte, neutrophil and eosinophil blood count. However, all of these variables were associated with hypoxemia (Table 2 ). The cut-off of $24 \mathrm{pg} / \mathrm{mL}$ for IL- 6 showed the best combination of sensitivity and specificity. In the group of all screened LTCF residents, baseline IL- 6 concentration > $24 \mathrm{pg} / \mathrm{mL}$ predicted the development of hypoxemia with a sensitivity of $88 \%$ and specificity of $89 \%$. Positive 
Table 1 Baseline characteristics of patients. Variables are provided as median (25th percentile, 75th percentile)

\begin{tabular}{|c|c|c|c|c|}
\hline & $\begin{array}{l}\text { No hypoxemia requiring } \\
\text { oxygen therapy }(n=26)\end{array}$ & $\begin{array}{l}\text { Hypoxemia requiring } \\
\text { oxygen therapy }(n=19)\end{array}$ & p (Mann-Whitney) & Cohen d \\
\hline Age (years) & $81(73,87)$ & $87(80.5,90)$ & 0.056 & 0.522 \\
\hline $\mathrm{SpO} 2$ & $0.96(0.95,0.97)$ & $0.91(0.86,0.94)$ & $<0.0001$ & 1.563 \\
\hline $\mathrm{CCl}$ & $5(4,6)$ & $7(6,8)$ & $<0.05$ & 1.038 \\
\hline CRP (mg/L) & $8.92(3.223,17.943)$ & $70.69(29.59,142.46)$ & $<0.0001$ & 1.398 \\
\hline IL-6 (pg/mL) & $12.3(7.3,20.5)$ & $43.1(26.3,116.7)$ & $<0.0001$ & 1.880 \\
\hline D-dimer (mg/L) & $1.215(0.558,2.625)$ & $1.58(0.78,3.43)$ & 0.198 & 0.366 \\
\hline Fibrinogen ( $g / L)$ & $3.6(3.3,4.18)$ & $4.45(3.825,5.825)$ & $<0.05$ & 0.869 \\
\hline Procalcitonine (ng/mL) & $0.02(0.02,0.03)$ & $0.132(0.048,0.313)$ & $<0.0001$ & 1.791 \\
\hline Ferritin (ug/L) & $175.9(94.13,429.3)$ & $295.18(149.79,778.02)$ & 0.125 & 0.465 \\
\hline AST (ukat/L) & $0.355(0.29,0.533)$ & $0.755(0.403,1.12)$ & $<0.0001$ & 1.214 \\
\hline ALT (ukat/L) & $0.25(0.16,0.37)$ & $0.35(0.26,0.54)$ & $<0.05$ & 0.613 \\
\hline Sodium (mmol/L) & $140.9(137.45,142.675)$ & $139(133.6,145.6)$ & 0.830 & 0.06 \\
\hline Potasium (mmol/L) & $4.09(3.85,4.36)$ & $3.71(3.235,4.503)$ & 0.184 & 0.386 \\
\hline Glucose $(\mathrm{mmol} / \mathrm{L})$ & $4.8(4.2,5.4)$ & $5.9(5.1,7.1)$ & $<0.01$ & 0.861 \\
\hline Urea (mmol/L) & $6.2(5.4,7.5)$ & $11(6,21.5)$ & $<0.01$ & 0.794 \\
\hline Creatinine (umol/L) & $74.5(59.25,101.25)$ & $110.9(73,264)$ & $<0.05$ & 0.729 \\
\hline Total bilirubin (umol/L) & $8.75(6.55,11.75)$ & $11.95(9.8,15.4)$ & $<0.0001$ & 0.728 \\
\hline WBC (cells/mL) & $4880(4100,6515)$ & $5640(4420,8640)$ & 0.167 & 0.395 \\
\hline LBC (cells/mL) & $1565(1045,2083)$ & $860(580,1300)$ & $<0.0001$ & 1.035 \\
\hline NBC (cells/mL) & $2680(2015,3800)$ & $3890(2950,7450)$ & $<0.05$ & 0.816 \\
\hline MBC (cells/mL) & $490(370,615)$ & $440(250,680)$ & 0.712 & 0.107 \\
\hline EBC (cells/mL) & $110(30,190)$ & $10(0,30)$ & $<0.05$ & 1.373 \\
\hline BBC (cells/mL) & $20(10,37)$ & $10(10,20)$ & 0.155 & 0.398 \\
\hline
\end{tabular}

$A L T$ alanine aminotransferase, $A S T$ aspartate aminotransferase, $B B C$ basophil blood count, $C C$ Charlson Comorbidity Index, $C R P C$-reactive protein, $E B C$ eosinophil blood count, IL-6 interleukin 6, $L B C$ lymphocyte blood count, $M B C$ monocyte blood count, NBC neutrophil blood count, $p$ probability, SpO2 oxygen saturation, WBC white blood cell blood count

predictive value (PPV) was $83 \%$, and negative predictive value was (NPV) of $93 \%$. After excluding the 7 patients diagnosed with severe Covid-19 and transferred to the hospital after the initial assessment, baseline IL-6 concentration over $24 \mathrm{pg} / \mathrm{mL}$ predicted the development of hypoxemia during the daily monitoring in the LTCF with the sensitivity of $100 \%$, specificity 89 , PPV of $77 \%$, and NPV 100\% (Table 3). Baseline CRP concentration with cut-off of $24 \mathrm{mg} / \mathrm{L}$ showed sensitivity and specificity inferior to IL-6 (Table 4). In multivariate analysis, baseline IL-6 concentration $>24 \mathrm{pg} / \mathrm{mL}$ was positively associated with the risk of hypoxemia development during follow up in LTCF residents after adjustment for CRP, age, gender, and glomerular filtration rate (Table 5).

\section{Discussion}

Our retrospective data analysis evaluated the potential role of biomarkers of inflammation, IL-6, CRP, procalcitonin, D-dimer and fibrinogen in the prediction of severe disease and need for oxygen therapy in residents of LTCF during Covid-19 outbreak. It suggests that IL-6 is a most robust predictor of hypoxemia requiring oxygen therapy. The concentration of IL- $6>24 \mathrm{pg} / \mathrm{mL}$ at the initial assessment is showing the best combination of sensitivity and specificity in predicting the hypoxemia requiring oxygen therapy.

There is a substantial body of evidence linking the IL6 concentration to the severity of disease and unfavorable outcome of Covid-19 [13, 14, 16-18, 20-23]. However, to our knowledge, this study is the first study which evaluated the ability of IL- 6 to predict the need for supplementary oxygen administration as a surrogate marker of severe Covid-19 requiring hospital admission in the population of high risk elderly patients. It also suggests clinical implications for intervention procedures for Covid-19 outbreak in LTCF. Han et al. examined the predictive value of various cytokines and concluded that IL-6 is the best predictor of severe Covid-19 [14]. A metanalysis of 9 studies concluded that increased IL- 6 is highly associated with severe disease. In this study, patients with severe Covid-19 had mean IL-6 $58 \mathrm{pg} / \mathrm{mL}$ compared to $17 \mathrm{pg} / \mathrm{mL}$ in mild disease [22]. A study by 
Table 2 AUC and optimal cut-offs for evaluated markers of hypoxemia requiring oxygen therapy by receiver operating characteristic curve analysis

\begin{tabular}{llll}
\hline marker & AUC & $\mathbf{9 5 \%} \mathbf{C l}$ of AUC & Asymptotic significance \\
\hline IL-6 & 0.911 & $0.819-1$ & 0.0001 \\
CRP & 0.887 & $0.777-0.996$ & 0.0001 \\
Fibrinogen & 0.788 & $0.640-0.936$ & 0.001 \\
D-dimer & 5.777 & $0.409-0.745$ & 0.392 \\
PCT & 0.886 & $0.767-1$ & 0.0001 \\
Total bilirubin & 0.712 & $0.535-0.888$ & 0.036 \\
Creatinine & 0.644 & $0.443-0.846$ & 0.152 \\
AST & 0.838 & $0.701-0.975$ & 0.001 \\
ALT & 0.675 & $0.491-0.858$ & 0.083 \\
LBC & 0.777 & $0.642-0.912$ & 0.001 \\
NBC & 0.728 & $0.582-0.783$ & 0.007 \\
EBC & 0.841 & $0.730-0.953$ & 0.0001
\end{tabular}

ALT alanine aminotransferase, $A S T$ aspartate aminotransferase, $A U C$ area under curve, 95\% Cl 95\% confidence interval, CRP C-reactive protein, EBC eosinophil blood count, IL-6 interleukin 6, LBC lymphocyte blood count, $N B C$ neutrophil blood count, $P C T$ procalcitonine, Asymptotic significance below 0.5 is regarded as statistically significant

Herold et al. found that IL- $6>80 \mathrm{pg} / \mathrm{mL}$ the predicts respiratory failure and need for mechanical ventilation in Covid-19. Like in our study, IL-6 was superior to CRP in ROC analysis. Chen et al. found cut-off $80 \mathrm{pg} / \mathrm{mL}$ of IL6 differentiates the survivors from the non-survivors [23]. We suggest that we found much lower optimal cutoff value (24 pg/mL compared to $80 \mathrm{pg} / \mathrm{mL}$ ) because we choose less severe endpoint (need for supplementary oxygen). Overall, we can conclude that our study is in concordance with previous studies and adds new aspects to the known body of evidence.

\section{Clinical significance and implications}

The residents of LTCF are one of the most vulnerable populations of the Covid-19 pandemic [8-10]. The outbreak of Covid-19 in LTCF might significantly burden

Table 3 Test evaluation of baseline concentration of IL-6 > 24 $\mathrm{pg} / \mathrm{mL}$ for predicting the development of hypoxemia requiring oxygen therapy during follow up after excluding patients admitted to hospital during initial triage

\begin{tabular}{lll}
\hline Statistic & Value & $\mathbf{9 5 \%}$ confidence interval \\
\hline Sensitivity & $100.00 \%$ & $69.15-100.00 \%$ \\
Specificity & $88.89 \%$ & $70.84-97.65 \%$ \\
Disease prevalence & $27.03 \%$ & $13.79-44.12 \%$ \\
PPV & $76.92 \%$ & $53.42-90.64 \%$ \\
NPV & $100.00 \%$ & Non available \\
Accuracy & $91.89 \%$ & $78.09-98.30 \%$ \\
\hline
\end{tabular}

95\% Cl 95\% confidence interval, NPV negative predictive value, PPV positive predictive value
Table 4 Test evaluation of baseline concentration of CRP $>24$ $\mathrm{mg} / \mathrm{L}$ for predicting the development of hypoxemia requiring oxygen therapy during follow up after excluding patients admitted to hospital during initial triage

\begin{tabular}{lll}
\hline Statistic & Value & $\mathbf{9 5 \%}$ Cl \\
\hline Sensitivity & $90.91 \%$ & 58.72 to $99.77 \%$ \\
Specificity & $79.41 \%$ & 62.10 to $91.30 \%$ \\
Disease prevalence & $24.44 \%$ & 12.88 to $39.54 \%$ \\
PPV & $58.82 \%$ & 41.84 to $73.94 \%$ \\
NPV & $96.43 \%$ & 80.52 to $99.44 \%$ \\
Accuracy & $82.22 \%$ & 67.95 to $92.00 \%$ \\
\hline
\end{tabular}

95\% Cl 95\% confidence interval, NPV negative predictive value, PPV positive predictive value

the local health care system. Timely and effective intervention is essential to reduce morbidity and mortality during such outbreak. According to the proposed guideline by Kim et al., the first phase of response should include broad testing a quick identification of cases and their clinical assessment and triage. In the next phase, monitoring of patients should be implemented in order to quickly identify the patients in need of hospital care [11]. Identification of patients at high risk of deterioration during the initial assessment may significantly improve the monitoring process by allocating the resources to high-risk patients more effectively. Especially in the case of limited human resources, the focus on high-risk patients is might contribute to mortality reduction and improve the overall outcome of the outbreak.

In our study, all patients that developed hypoxemia requiring oxygen therapy during the follow up had the baseline concentration of IL- 6 over $24 \mathrm{pg} / \mathrm{mL}$. As a screening tool, it provides excellent sensitivity with an acceptable specificity over $88 \%$. It may be used to identify the patients with a high risk of hypoxemia. It also identifies the patients with low risk for hypoxemia with great negative predictive value. This might help in the decision making for admission during the initial triage. Baseline CRP concentration with cut-off of $24 \mathrm{mg} / \mathrm{L}$ showed sensitivity and especially specificity inferior to

Table 5 Multivariate binary logistic regression analysis of the association of IL-6>24 pg/mL, CRP, glomerular filtration rate, gender, and age with the probability of development of hypoxemia requiring oxygen therapy during follow up

\begin{tabular}{llll}
\hline & $\mathbf{p}$ & OR & $\mathbf{9 5 \%} \mathbf{C l}$ of $\mathbf{~ O R}$ \\
\hline $\mathrm{IL}-6>24 \mathrm{pg} / \mathrm{mL}$ & $<0.05$ & 39.741 & $1.838-859.426$ \\
CRP & 0.203 & 1.028 & $0.985-1.072$ \\
age & 0.187 & 1.133 & $0.943-1.364$ \\
gender (male) & 0.175 & 0.088 & $0.003-2.944$ \\
GFR & 0.773 & 0.979 & $0.850-1.128$ \\
\hline
\end{tabular}

CRP C-reactive protein, GFR glomerular filtration rate, 95\% Cl 95\% confidence interval, $I L-6$ interleukin $6, O R$ odds ratio, $p$ probability 
IL-6, however, might be used as cheaper alternative in low resource setting.

For the purposes of our study, we defined that the residents that developed hypoxemia requiring oxygen therapy are the cases that needed close monitoring and early transfer to the hospital. The rationale is that patients who developed hypoxemia requiring oxygen therapy are those that might benefit from the early pharmacological treatment in order to reduce mortality. According to the body of evidence on remdesivir and dexamethasone, these drugs significantly improves the outcome in the patients needing conventional oxygen therapy [24, 25]. Thus, the patients with early disease but in the high risk of development of hypoxemia are those patients who will profit from close observation and rapid initiation of remdesivir and dexamethasone in case of progression of Covid-19.

\section{IL-6 and development of severe Covid-19}

IL-6 is produced by stromal cells and virtually all immune system cells in the lungs and its secretion is stimulated by proinflammatory cytokines, especially interleukin $1 \beta$ (IL-1 $\beta$ ) and tumor necrosis factor $\alpha$ (TNF $\alpha)$. In the early stages of the infection, it is produced by lung macrophages after stimulation of toll-like receptors [26]. An important trait of IL-6 upregulation in Covid-19 is that it precedes the development of acute lung injury that implicates its usability as an early marker of severe disease [22]. However, there is controversy if excessive IL-6 synthesis is true a cornerstone of the pathogenesis of respiratory failure in Covid-19 or is just an epiphenomenon of increased IL-1 $\beta$ and TNF $\alpha$ in the cytokine storm [16]. Predominant theory is that overexpression of IL- 6 have a crucial role in the incitement and propagation of the so-called cytokine storm leading to lung injury and ARDS [16, 17]. It is believed that IL-6 increases the permeability of lung capillaries driving the ARDS development and also stimulates the coagulation pathway leading to microthrombi in lung circulation and increases the risk of thrombotic event [26]. A study by Giamarellos-Bourboulis et al. suggests that patients with severe respiratory failure in Covid-19 suffer from distinct types of immune dysregulation which are mediated by IL- 6 upregulation. This dysregulation is characterized by high production of proinflammatory cytokines by monocytes and macrophages and CD4 lymphocyte depletion that contributes to the progression of inflammation of lung parenchyma [17]. The direct role of IL-6 in Covid-19 pathogenesis is further supported by findings that IL- 6 inhibition improves the prognosis of severe Covid-19 [26, 27].

\section{Other markers of severe disease}

In our study, patients who developed hypoxemia had significantly higher serum concentrations of AST,
ALT, CRP, serum glucose, creatinine, procalcitonin, and fibrinogen. They also had significantly higher blood count of neutrophils and lower count of lymphocytes and eosinophils. These variables were identified as markers of severe disease by the previous studies [13]. Medians of other well-established markers of serious disease, D-dimer and ferritin were higher in patients who developed hypoxemia, however, the differences were not statistically significant. Using ROC analysis, we concluded that IL-6 is better marker of hypoxemia than CRP or other evaluated variables. Other proinflammatory cytokines, like IL-1 $\beta$ or TNF- $\alpha$ are also associated with severity of Covid19 and might also serve as biomarkers [28, 29]. However, because the primary goal of our study was to improve the practical algorithm for intervention in LTCF, we focused on examination of biomarkers that are well established in clinical practice and are available for evaluation in most commercial biochemical laboratories in Slovakia.

\section{Prognosis of LTCF residents suffering from Covid-19}

According to an epidemiologic study by McMichael et al., during the outbreak in one LTCF in Washington, USA, $54.5 \%$ of residents required hospitalization and required hospital admission and $33.7 \%$ of infected residents died [8]. In our study, we identified 59 residents with positive swabs for SARS-CoV-2. Of these patients. Thirty-two patients (54\%) were admitted to hospital which is similar to the study by McMichael et al. In our study, 13 patients (22\%) died. That is less than in the study by McMichael et al., however, this difference of proportion might be attributed to potentially different age and comorbidity status of residents.

\section{Limitations}

The limitations of our study are the relatively low sample size and retrospective design. Larger prospective studies are needed to obtain more robust data and to evaluate if the examination of IL- 6 during the initial assessment leads to better prognosis of LTCF residents and improves the management of the Covid-19 outbreaks in the LTCFs.

Because of the retrospective design of the study, there might be a concern of bias in the sensitivity of the diagnosis of hypoxemia between groups of hospitalized and outpatient residents. The hospitalized patients were naturally more closely monitored and therefore might be more likely to be diagnosed with hypoxemia. However, we regard this potential bias as insignificant because the outpatient residents were daily monitored for the symptoms and signs of respiratory failure, and patients suffering from dyspnea 
and patients with tachypnea and/or $\mathrm{SpO} 2$ below $90 \%$ were transferred to hospital. In normoxic patients, the bias of pulse oximetry comparing to $\mathrm{SaO} 2$ is regarding to be insignificant. It reliably identifies the patients with $\mathrm{SaO} 2$ below $90 \%$ and is a reliable screening tool for hypoxemia with very high negative predictive value [30].

Another limitation is that we did not evaluate the IL-1 $\beta$ as a possible biomarker of hypoxemia. IL-1 $\beta$ is believed to contribute to the cytokine storm in Covid-19. It is the cytokine which stimulates the IL- 6 production and therefore is on the level above the IL- 6 in the cascade of cytokine storm [28]. The theory that IL-1 $\beta$ have an important role in the pathogenesis of Covid-19 is widely accepted [29]. However, recent study by Mandel et al. found that baseline concentration of IL- $1 \beta$ is not significantly higher in non-survivors [18]. Giamarellos-Bourboulis et al. concluded that immune dysregulation in severe Covid-19 is directly driven by IL-6, not IL-1 $\beta$ upregulation [17].

Our study included more females than males. This proportion reflects the LTCF population in which female to male ratio was three to one and is not a result of deliberate selection.

\section{Conclusions}

Baseline IL-6 concentrations over $24 \mathrm{pg} / \mathrm{mL}$ in LTCF residents suffering from Covid-19 predicts the development of hypoxemia requiring oxygen therapy with excellent sensitivity and good specificity. Patients with the IL-6 above $24 \mathrm{pg} / \mathrm{m}$ at initial assessment seems to be at high risk of development of respiratory failure and might benefit from early hospitalization and close follow-up. Further studies are needed to evaluate the real benefits of systematic examination of IL- 6 during the initial assessment and its use as predictive factor of severe disease.

\begin{abstract}
Abbreviations
ALT: Alanine aminotransferase; ARDS: Acute respiratory distress syndrome; AST: Aspartate aminotransferase; AUC: Area under curve; BBC: Basophil blood count; CCl: Charlson Comorbidity Index; Covid-19: Coronavirus disease 2019; EBC: Eosinophil blood count; IL-6: Interleukin 6; IL-1B: Interleukin 13; LBC: Lymphocyte blood count; LTCF: Long term care facility; MBC: Monocyte blood count; NBC: Neutrophil blood count; NPV: Negative predictive value; p: probability; PPV: Positive predictive value; ROC: Receiver operating characteristic curve; SARS-CoV-2: Severe acute respiratory distress syndrome coronavirus 2; SaO2: Oxygen saturation from arterial blood; SpO2: Oxygen saturation by pulse oximetry; TNF-a: Tumor necrosis factor a; WBC: White blood cell blood count
\end{abstract}

\section{Supplementary Information}

The online version contains supplementary material available at https://doi. org/10.1186/s12879-021-05945-8.

Additional file 1: Supplementary table: Clinical characteristics, major comorbidities, major complications and most significant biochemical and blood count variables provided for individual patients.

\section{Acknowledgments}

The authors would like to thank all personnel of the University Hospital in Bratislava that contributed to the intervention in LTCF and to this study.

\section{Authors' contributions}

PS drafted the manuscript, co-developed the study design, and participated in the data gathering and analysis and interpretation of results. AK co-drafted the manuscript, planned the intervention, developed the study design, and participated in the data gathering and analysis and interpretation of results. $\mathrm{JH}$ planned the intervention and participated in the data gathering and analysis and interpretation of results. IS and RL co-drafted the manuscript and participated in the data gathering and analysis and interpretation of results. MK, BK, and AK participated in data gathering and analysis and interpretation of results. All authors have read and approved the final manuscript.

\section{Funding}

The research was supported by Slovak Agency for Research and Development No: APW-18-0287.

\section{Availability of data and materials}

The dataset used and analyzed during this study is available as a supplementary material.

\section{Declarations}

\section{Ethics approval and consent to participate}

This study was carried out in concordance with The Code of Ethics of the World Medical Association (Declaration of Helsinki) for experiments involving humans and was approved by the local Ethical Committee of University Hospital in Bratislava. Written informed consent for participation was obtained from all participants before enrolment to the study. No administrative permission to access the raw data used in this study was required by local authorities, hospital or LTCF. The raw data were fully anonymized before its use. The investigators preserved the full anonymity of all participants.

\section{Consent for publication}

Not applicable.

\section{Competing interests}

The authors declare that they have no competing interests.

\section{Author details}

'Department of Infectology and Geographical Medicine, Faculty of Medicine, Comenius University in Bratislava, Bratislava, Slovakia. ${ }^{2}$ Department of Infectology and Geographical Medicine, Faculty of Medicine, Slovak Medical University, Bratislava, Slovakia. ${ }^{3}$ 2nd Department of Neurology, Faculty of Medicine, Comenius University in Bratislava, Bratislava, Slovakia. ${ }^{4}$ Emergency Department, University Hospital in Bratislava, Bratislava, Slovakia. ${ }^{5}$ Institute of Molecular Biomedicine, Faculty of Medicine, Comenius University in Bratislava, Bratislava, Slovakia. ${ }^{6}$ Institute of Physiology, Faculty of Medicine, Comenius University in Bratislava, Bratislava, Slovakia.

Received: 13 July 2020 Accepted: 1 March 2021

Published online: 29 March 2021

\section{References}

1. Wu Z, McGoogan JM. Characteristics of and important lessons from the coronavirus disease 2019 (COVID-19) outbreak in China: summary of a report of 72314 cases from the Chinese Center for Disease Control and Prevention. JAMA. 2020. https://doi.org/10.1001/jama.2020.2648 [Epub ahead of print].

2. World Health Organization. Rolling updates on coronavirus disease (COVID19). 2020, https://www.who.int/emergencies/diseases/novel-coronavirus-201 9/events-as-they-happen. opens in new tab, (Accesed 18 May 2020).

3. Zhou F, Yu T, Du R, et al. Clinical course and risk factors for mortality of adult inpatients with COVID-19 in Wuhan, China: a retrospective cohort study. Lancet. 2020;395(10229):1054-62. https://doi.org/10.1016/S0140-673 6(20)30566-3.

4. Russell TW, Hellewell J, Jarvis Cl, et al. Estimating the infection and case fatality ratio for coronavirus disease (COVID-19) using age-adjusted data 
from the outbreak on the Diamond Princess cruise ship, February 2020. Euro Surveill. 2020;25(12):2000256. https://doi.org/10.2807/1560-7917.ES.202 0.25 .12 .2000256

5. Rathore V, Galhotra A, Pal R, Sahu KK. COVID-19 pandemic and children: a review. J Pediatr Pharmacol Ther. 2020;25(7):574-85. https://doi.org/10. 5863/1551-6776-25.7.574

6. Allotey J, Stallings E, Bonet $\mathrm{M}$, et al. Clinical manifestations, risk factors, and maternal and perinatal outcomes of coronavirus disease 2019 in pregnancy: living systematic review and meta-analysis. BMJ. 2020;370:m3320. Published 2020 Sep 1. https://doi.org/10.1136/bmj.m3320.

7. Verity R, Okell L, Dorigatti I, et al. Estimates of the severity of coronavirus disease 2019: a model-based analysis. Lancet. 2020;20(6):669-77. https://doi. org/10.1016/S1473-3099(20)30243-7.

8. Lai CC, Wang JH, Ko WC, et al. COVID-19 in long-term care facilities: an upcoming threat that cannot be ignored. J Microbiol Immunol Infect. 2020; 53(3):444-6. https://doi.org/10.1016/j.jmii.2020.04.008.

9. Abrams HR, Loomer L, Gandhi A, Grabowski DC. Characteristics of U.S. Nursing Homes with COVID-19 Cases. J Am Geriatr Soc. 2020. https://doi. org/10.1111/jgs.16661 [published online ahead of print, 2020 Jun 2].

10. McMichael TM, Currie DW, Clark S, et al. Epidemiology of Covid-19 in a long-term Care Facility in King County, Washington. N Engl J Med. 2020; 382(21):2005-11. https://doi.org/10.1056/NEJMoa2005412.

11. Kim G, Wang M, Pan H, et al. A health system response to covid-19 in longterm care and post-acute care: a three-phase approach. J Am Geriatr Soc. 2020. https://doi.org/10.1111/jgs.16513 [published online ahead of print, 2020 Apr 28].

12. Ponti G, Maccaferri M, Ruini C, Tomasi A, Ozben T. Biomarkers associated with COVID-19 disease progression. Crit Rev Clin Lab Sci. 2020:1-11. https:// doi.org/10.1080/10408363.2020.1770685 [published online ahead of print, 2020 Jun 5].

13. Velavan TP, Meyer CG. Mild versus severe COVID-19: laboratory markers. Int $J$ Infect Dis. 2020;95:304-7. https://doi.org/10.1016/j.ijid.2020.04.061.

14. Herold T, Jurinovic V, Arnreich C, et al. Elevated levels of IL-6 and CRP predict the need for mechanical ventilation in COVID-19. J Allergy Clin Immunol. 2020;146(1):128-136.e4. https://doi.org/10.1016/j.jaci.2020.05.008.

15. Zeng F, Huang $Y$, Guo $Y$, et al. Association of inflammatory markers with the severity of COVID-19: a meta-analysis. Int J Infect Dis. 2020;96:467-74. https://doi.org/10.1016/ji.ijid.2020.05.055.

16. Magro G. SARS-CoV-2 and COVID-19: is interleukin-6 (IL-6) the 'culprit lesion' of ARDS onset? What is there besides Tocilizumab? SGP130Fc. Cytokine $X$ 2020;2(2):100029. https://doi.org/10.1016/j.cytox.2020.100029 [published online ahead of print, 2020 May 14].

17. Giamarellos-Bourboulis EJ, Netea MG, Rovina N, et al. Complex Immune Dysregulation in COVID-19 Patients with Severe Respiratory Failure. Cell Host Microbe. 2020;27(6):992-1000.e3. https://doi.org/10.1016/j.chom.2020.04.009.

18. Mandel M, Harari G, Gurevich M, Achiron A. Cytokine prediction of mortality in COVID19 patients [published online ahead of print, $2020 \mathrm{Jul} 10]$. Cytokine. 2020;134:155190. https://doi.org/10.1016/j.cyto.2020.155190.

19. Han H, Ma Q, Li C, et al. Profiling serum cytokines in COVID-19 patients reveals IL-6 and IL-10 are disease severity predictors. Emerg Microbes Infect. 2020:9(1):1123-30. https://doi.org/10.1080/22221751.2020.1770129.

20. Liu T, Zhang J, Yang Y, et al. The role of interleukin-6 in monitoring the severe case of coronavirus disease 2019. EMBO Mol Med. 2020:e12421. https://doi.org/10.15252/emmm.202012421 [published online ahead of print, 2020 May 19].

21. Ulhaq ZS, Soraya GV. Interleukin-6 as a potential biomarker of COVID-19 progression. Med Mal Infect. 2020;50(4):382-3. https://doi.org/10.1016/j. medmal.2020.04.002.

22. Aziz M, Fatima R, Assaly R. Elevated interleukin-6 and severe COVID-19: A meta-analysis. J Med Virol. 2020. https://doi.org/10.1002/jmv.25948 [published online ahead of print, 2020 Apr 28]

23. Chen T, Wu D, Chen H, et al. Clinical characteristics of 113 deceased patients with coronavirus disease 2019: retrospective study. BMJ. 2020;m1091:368

24. Beigel JH, Tomashek KM, Dodd LE, et al. Remdesivir for the Treatment of Covid-19 - Preliminary Report. N Engl J Med. 2020. https://doi.org/10.1056/ NEJMoa2007764 [published online ahead of print, 2020 May 22].

25. Horby P, Shen Lim W, Emberson J, et al. Effect of dexamethasone in hospitalized patients with COVID-19: preliminary report. medRxiv. 2020. https://doi.org/10.1101/2020.06.22.20137273.

26. Hunter CA, Jones SA. IL-6 as a keystone cytokine in health and disease. Nat Immunol. 2015;16:448-57.
27. Guaraldi G, Meschiari M, Cozzi-Lepri A, et al. Tocilizumab in patients with severe COVID-19: a retrospective cohort study. Lancet Rheumatol. 2020;2(8): e474-84. https://doi.org/10.1016/S2665-9913(20)30173-9.

28. Zhang C, Wu Z, Li JW, Zhao H, Wang GQ. Cytokine release syndrome in severe COVID-19: interleukin-6 receptor antagonist tocilizumab may be the key to reduce mortality. Int J Antimicrob Agents. 2020;55(5):105954. https:// doi.org/10.1016/j.jjantimicag.2020.105954Jubran A. Pulse oximetry. Crit Care. 2015;19(1):272. Published 2015 Jul 16. doi:10.1186/s13054-015-0984-8.

29. Conti P, Caraffa A, Gallenga CE, et al. IL-1 induces throboxane-A2 (TXA2) in COVID-19 causing inflammation and micro-thrombi: inhibitory effect of the IL-1 receptor antagonist (IL-1Ra). J Biol Regul Homeost Agents. 2020;34(4). https://doi.org/10.23812/20-34-4EDIT-65 [published online ahead of print, 2020 Aug 3].

30. Kohyama T, Moriyama K, Kanai R, et al. Accuracy of pulse oximeters in detecting hypoxemia in patients with chronic thromboembolic pulmonary hypertension. PLoS One. 2015;10(5):e0126979. https://doi.org/10.1371/journa I.pone.0126979 [published correction appears in PLoS One. 2015;10(10): e0141944] Published 2015 May 15.

\section{Publisher's Note}

Springer Nature remains neutral with regard to jurisdictional claims in published maps and institutional affiliations.

\section{Ready to submit your research? Choose BMC and benefit from:}

- fast, convenient online submission

- thorough peer review by experienced researchers in your field

- rapid publication on acceptance

- support for research data, including large and complex data types

- gold Open Access which fosters wider collaboration and increased citations

- maximum visibility for your research: over $100 \mathrm{M}$ website views per year

At $\mathrm{BMC}$, research is always in progress.

Learn more biomedcentral.com/submissions 